\title{
Methadone maintenance was more effective for treatment retention for opioid dependence than psychosocially enriched detoxification
}

Sees KL, Delucchi KL, Masson C, et al. Methadone maintenance vs 180-day psychosocially enriched detoxification for
treatment of opioid dependence. A randomized controlled trial.JAMA 2000 Mar 8;283:1303-10.

QUESTION: In adults with opioid dependence, do standard methadone maintenance (MM) and methadone assisted detoxification plus intensive psychosocial services lead to similar outcomes?

Source of funding:

National Institute on

Drug Abuse.

For correspondence:

$\operatorname{Dr} S$ M Hall,

Department of

Psychiatry, University

of California, San

Francisco, 401

Parnassus Avenue, Box

0984-TRC, San

Francisco, CA

94143-0984, USA.

Fax +14154767677

\section{Design}

Randomised \{allocation concealed*\}†, unblinded,* controlled trial with 12 month follow up.

\section{Setting}

A Veterans Affairs medical centre in San Francisco, California, United States.

\section{Patients}

179 non-veteran adults who were $\geqslant 18$ years of age (mean age 39 y, 59\% men); had a diagnosis of opioid dependence according to the Diagnostic and Statistical Manual of Mental Disorders, Revised Third Edition; and tested positive for opioid use and negative for methadone use. Exclusion criteria were contraindications to methadone treatment, psychiatric medical conditions that interfered with treatment, pregnancy, lactation, concurrent substance abuse treatment, no signs of opioid withdrawal on 3 occasions, methadone treatment in the previous week, participation in a follow up phase of a previous methadone detoxification research protocol, or inability to participate for 12 months. Follow up was $76 \%$ at 6 months and $75 \%$ at 12 months.

\section{Intervention}

Adults were allocated to $\mathrm{MM}(\mathrm{n}=91)$ for 14 months or to 120 days of methadone assisted maintenance and 60 days of methadone assisted detoxification with intensive psychosocial services and 8 months of aftercare (M180) $(\mathrm{n}=88)$. The initial methadone dose was $30 \mathrm{mg} /$ day, which was increased to $80 \mathrm{mg} /$ day within the first 3 treatment weeks. The maximum dose was $100 \mathrm{mg}$ /day, which was reached by day 44 .

\section{Main outcome measures}

The main outcome was treatment retention. The following outcomes were also assessed but had $<80 \%$ follow up: opioid and cocaine use (assessed by self report and urine screening test); HIV risk behaviors (Risk of AIDS Behavior scale score); and function in employment, drug use, alcohol use, legal, family, and psychiatric problem areas (Addiction Severity Index).

\section{Main results}

Analysis was by intention to treat. Patients in the MM group remained in treatment longer than patients in the M180 group (median 439 d, $95 \%$ CI 413 to 441 v 174 d, CI 161 to $181 ; \mathrm{p}<0.001)$. More patients were discharged from treatment for non-compliance in the M180 group than in the MM group (table).

\section{Conclusion}

In adults with opioid dependence, treatment retention was greater for methadone maintenance than for methadone assisted detoxification plus intensive psychosocial services.

*See glossary.

$\dagger$ Information provided by author.

Methadone maintenance (MM) v methadone assisted detoxification and psychosocial services (M180) for opioid dependence:

$\begin{array}{llll}\text { Outcome at } 1 \text { year } & \text { MM } & \text { M180 } & \text { RBI } \\ (95 \% & \mathrm{Cl}) & \text { NNT }(\mathrm{Cl})\end{array}$

Treatment retention $\quad 74 \% \quad 55 \% \quad 35 \%$ (8 to 71$) \quad 6(4$ to 20$)$

$\ddagger$ Abbreviations defined in glossary; $\mathrm{RBI}, \mathrm{NNT}$, and $\mathrm{Cl}$ calculated from data in article.

\section{COMMENTARY}

In this study by Sees $e t a l, \mathrm{MM}$ is compared with a detoxification package involving an identical methadone dose followed by detoxification and aftercare (M180). MM resulted in better treatment retention than did M180 and lowered HIV risk and legal status scores, which is no doubt a reflection of increased illicit drug use in M180. Treatment retention in the M180 group was adversely affected by receipt of an intensive psychosocial intervention and rapid opiate detoxification in a context where most patients did not want to detoxify.

M180 had about $10 \%$ to $15 \%$ more discharges than did MM during the first 4 months (even though the medication used during this period was identical in both groups), presumably because of the more stringent attendance requirements (1.25 $v 3$ to 4 hours per wk). The detoxification phase in M180 was 60 days and involved a weekly $10 \mathrm{mg}$ methadone reduction from an average starting dose of 85 $\mathrm{mg}$ methadone, which is likely to have generated prolonged mild to moderate opiate withdrawal symptoms. Only $50 \%$ of patients in this group reported wanting to stop illicit opiate use in addition to their methadone, and no doubt even fewer would have wanted complete opioid detoxification.

Failure to attend treatment sessions resulted in discharge, but continued use of heroin or other illicit drugs did not. Indeed, $60 \%$ to $70 \%$ of participants continued to use illicit opiates (even when on maximal methadone treatment in both groups), which increased to about $90 \%$ after methadone termination in the M180 group. $50 \%$ to $70 \%$ of patients also continued to use cocaine during the study. These high rates suggest that the psychosocial intervention was insufficiently targeted at this clinically important goal.

For clinicians, this study shows the importance of targeting illicit drug use with a psychosocial intervention, the dangers of compulsory intensive psychosocial treatments, and the necessity that those who undergo therapeutic detoxification must really want to do so and are prepared to tolerate the level of suffering required.

Fergus Law, MBChB University of Bristol Bristol, $U K$ 